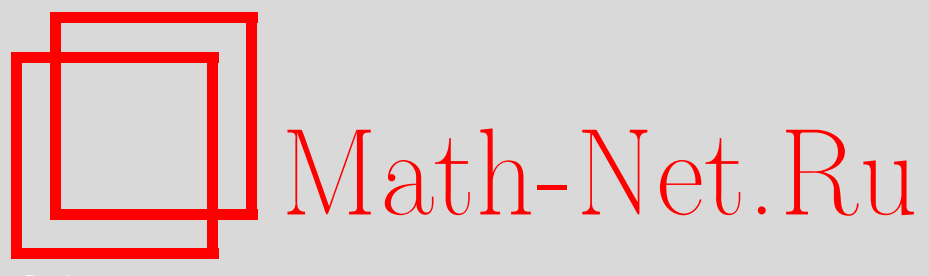

С. М. Асеев, В. М. Бухштабер, Р. И. Григорчук, В. З. Гринес, Б. М. Гуревич, А. А. Давыдов, А. Ю. Жиров, Е. В. Жужома, М. И. Зеликин, А. Б. Каток, А. В. Клименко, В. В. Козлов, В. П. Лексин, М. И. Монастырский, А. И. Нейштадт, С. П. Новиков, Е. А. Сатаев, Я. Г. Синай, А. М. Стёпин, Дмитрий Викторович Аносов (некролог), УМН, 2015, том 70, выпуск 2, 181-191

DOI: https://doi.org/10.4213/rm9652

Использование Общероссийского математического портала Math-Net.Ru подразумевает, что вы прочитали и согласны с пользовательским соглашением http://www . mathnet.ru/rus/agreement

Параметры загрузки:

IP: 54.162 .27 .143

26 апреля 2023 г., 02:57:46

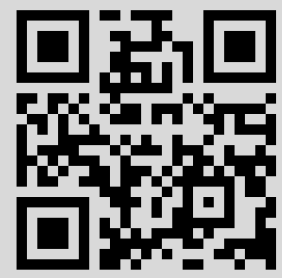




\section{Дмитрий Викторович Аносов}

5 августа 2014 г. скончался выдающийся математик, специалист в области дифференциальных уравнений, динамических систем и смежных вопросов геометрии и топологии, академик, советник РАН, главный научный сотрудник Математического института им. В. А. Стеклова РАН, заслуженный профессор МГУ им. М. В. Ломоносова Дмитрий Викторович Аносов.

Многообразная деятельность Д.В. Аносова снискала ему всемирную известность в математическом сообществе. Имя Аносова навсегда связано с одним из основных разделов теории динамических систем - гиперболической динамикой. Его исследования сыграли определяющую роль в формировании и развитии идеи глобальной гиперболичности в динамике, в разработке всей системы соответствующих понятий и методов. Это привело к новому взаимовлиянию теории дифференциальных уравнений,

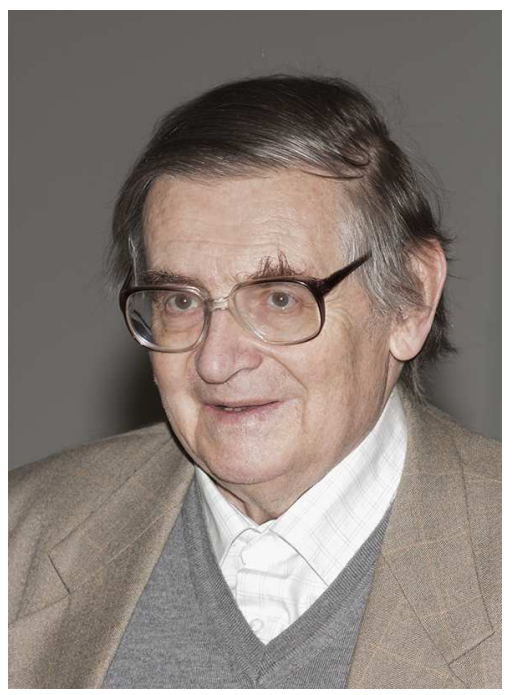
теории динамических систем, механики, геометрии, топологии и их приложений.

Дмитрий Викторович родился в Москве 30 ноября 1936 г. Его родители Виктор Яковлевич Аносов и Нина Константиновна Воскресенская работали в Институте общей и неорганической химии. Он стал студентом мехмата МГУ в год, когда Московский университет переехал в новое здание на Ленинских горах. Направление своих интересов Д. В. Аносов определил уже на первом курсе, его научными руководителями стали Л. С. Понтрягин и Е. Ф. Мищенко. На старших курсах он посещал спецкурс и семинар А. Н. Колмогорова по теории динамических систем и семинар М. М. Постникова и А. С. Шварца по алгебраической топологии.

Под влиянием Понтрягина еще студентом Аносов изучил некоторые главы книги А. А. Андронова, А. А. Витта (правда, в первом издании этот автор не был указан) и С. Э. Хайкина "Теория колебаний". "Они произвели на меня исключительно сильное впечатление, - пожалуй, ни одна книга впоследствии такого впечатления не производила", - писал Дмитрий Викторович в статье [31].

Одним из главных результатов, полученных еще в 1937 г. Андроновым и Понтрягиным, было введение понятия грубой (структурно устойчивой) динамической системы и установление следующего критерия: поток в ограниченной области плоскости груб тогда и только тогда, когда он имеет лишь конечное множество состояний равновесия и периодических траекторий, причем все они гиперболические и нет сепаратрис, идущих из седла в седло. По свидетельству сокурсников, юношеской мечтой Д. В. Аносова была разработка теории грубых систем. Его достижениям в этом и других направлениях теории динамических систем и дифференциальных уравнений посвящена статья в УМH, 52:2(314) (1997), 193-200, написанная к 60-летию со

DOI: $10.4213 / \mathrm{rm} 9652$ 
дня рождения Дмитрия Викторовича. Здесь мы дополним тот обзор математического творчества Д.В. Аносова, проследим связи работ, выполненных им после 1996 г., с фундаментальными результатами, полученными им ранее, и кратко очертим современное развитие его идей.

Первые работы Д. В. Аносова посвящены задачам теории устойчивости, сингулярным возмущениям и методу усреднения для дифференциальных уравнений. В его статье о предельных циклах (1960 г.) рассматривались системы вида

$$
\dot{x}=f(x, y, \varepsilon), \quad \dot{y}=\varepsilon g(x, y, \varepsilon)
$$

класса $C^{1}$. Здесь $\varepsilon$ - малый положительный параметр, вектор $y$ называется медленной переменной, а $x$ - быстрой. Система для $x$ при $y=$ const называется быстрой системой. Д. В. Аносов изучал вопрос о существовании у системы (1) периодического решения, близкого к невырожденному периодическому решению $L_{0}$ предельной (при $\varepsilon \rightarrow 0)$, так называемой вырожденной, системы

$$
\dot{y}=\varepsilon g(\varphi(y), y, 0),
$$

где $\varphi(y)$ - гладко зависящее от $y$ положение равновесия быстрой системы. Существование таких решений Д. В. Аносов доказал при условии, что собственные значения матрицы $\left(\partial f_{i} / \partial x_{j}\right)$ в точках $L_{0}$ не лежат на мнимой оси (несколько ранее подобный результат для периодических систем класса $C^{2}$ был получен Л. Флатто и Н. Левинсоном). В статье был приведен пример, показывающий, что сделанное предположение о собственных значениях существенно; кроме того, в этом примере периодические решения вблизи $L_{0}$ существуют для большинства $\varepsilon$. На Киевском симпозиуме по нелинейным колебаниям (1961 г.) В. Вазов высказал гипотезу о том, что для достаточно малых значений комплексного параметра $\varepsilon$, принадлежащих множеству плотности 1 в нуле, формальное решение системы (1) соответствует "настоящим" периодическим решениям. В 1996 г. Д. В. Аносов показал, что даже для аналитических систем с невырожденными периодическими решениями у предельной системы возможны как отсутствие периодических решений при всех $\varepsilon$, так и существование периодических решений для большинства $\varepsilon$, причем для них формальное решение не является асимптотическим разложением.

В следующей работе (1960 г.) Д. В. Аносов рассмотрел систему (1) с точки зрения метода усреднения. Формализм этого метода известен давно; он (хотя и без достаточного обоснования) успешно применялся к задачам небесной механики и теории колебаний. При $\varepsilon=0$ в системе (1) изменяется лишь $x$. При $\varepsilon \neq 0$ задача состоит в построении так называемой медленной системы

$$
\dot{\bar{y}}=\varepsilon B(\bar{y})
$$

приближенно описывающей эволюцию медленной переменной $y$.

Аносов впервые стал рассматривать систему (1) в самой общей постановке, когда $x$ - точка компактного многообразия $M$. Предполагается, что 1) быстрая система имеет инвариантную меру $\mu_{y}$ с гладкой плотностью, зависящей от $x, y$, и 2) быстрая система эргодична при почти всех значениях $y$. Правую часть $\varepsilon B(\bar{y})$ медленной системы Д.В. Аносов определяет как среднюю (по условной мере $\mu_{y}$ на $M$ ) скорость изменения $y$ в (1) (такую медленную систему называют усредненной). Для уклонения $\sup _{0<t<1 / \varepsilon}\left|y_{t}^{\varepsilon}-\bar{y}_{t}\right|$ он установил сходимость к нулю по мере; здесь $y_{t}^{\varepsilon}$ и $\bar{y}_{t}-$ медленная компонента решения системы (1) и решение усредненной системы соответственно с одинаковыми начальными (при $t=0$ ) значениями. Этот результат составил основу его кандидатской диссертации, выполненной под руководством Л. С. Понтрягина и защищенной в Московском университете летом 1961 г.

Как было отмечено выше, обоснование формализма метода усреднения отставало от его использования. Первый результат в этом направлении был получен П. Фату 
(1928 г.) в случае $M=\mathbb{T}^{1}$. Случай $M=\mathbb{T}^{n}, n \geqslant 2$, был рассмотрен Н. Н. Боголюбовым (1945 г.) в предположении, что быстрая система - иррациональная обмотка тора. Для гамильтоновых систем прогресс, приведший затем к теории КАМ и теории Н. Н. Нехорошева, связан с работой А. Н. Колмогорова (1954 г.). В развитие идей Д. В. Аносова возникли новые вопросы: об оценке меры исключительного множества (начальных данных, для которых метод усреднения не применим), о судьбе траекторий с начальными данными из этого множества, об оценке точности метода усреднения вне исключительного множества. В случае быстрых движений по компактному многообразию открылась возможность рассматривать задачу при более сильных статистических свойствах быстрой системы и получать улучшенные оценки погрешности метода. В этих направлениях дальнейшие исследования были выполнены В. И. Арнольдом, В. И. Бахтиным и Ю. И. Кифером; см. [43].

Наряду с защитой диссертации, содержащей новый подход к задаче об усреднении в системах дифференциальных уравнений с быстро колеблющимися решениями и основополагающий результат в рамках этого подхода, в 1961 г. произошло еще одно знаменательное событие в жизни Д. В. Аносова: осенью он поехал в Киев для участия в уже упомянутом выше Международном симпозиуме по нелинейным колебаниям. Вот что пишет Дмитрий Викторович в [31]: "Киевляне издали к началу конференции тезисы ряда докладов в виде отдельных брошюрок (тогда это требовало заметных усилий), причем тезисы иностранцев были переведены на русский язык. И вот, стоя в очереди регистрирующихся участников и заглядывая через плечи вперед, я стал рассматривать стопки этих брошюрок и прочитал название одной из них: $C$. Cмейл. Структурно устойчивый дифферениируемый гомеоморфизм с бесконечным числом периодических точек. В этот момент мир для меня перевернулся и началась новая жизнь".

После Киевского симпозиума Смейл приехал в Москву, встретился в Математическом институте им. В. А. Стеклова с Д. В. Аносовым, В. И. Арнольдом, С. П. Новиковым, Я. Г. Синаем и подробнее, чем после доклада на симпозиуме, рассказал свою конструкцию "подковы". Тогда же он высказал две гипотезы: о грубости гиперболического автоморфизма двумерного тора и геодезического потока на компактном римановом многообразии отрицательной кривизны. Уже в следующем году в ДАН СССР вышла статья Д. В. Аносова [1] о грубости геодезического потока, в которой отчетливо была выделена роль свойства гиперболичности как основной причины грубости динамической системы. Качественно свойство гиперболичности означает, что для любой фиксированной траектории поведение соседних траекторий по отношению к ней похоже на поведение траекторий возле седловой особой точки. Если у такой системы имеется последовательность точек, в которой каждая точка находится $\varepsilon$-близко к образу предыдущей ( $\varepsilon$-псевдотраектория), то вблизи этой последовательности существует (и единственна) настоящая траектория системы. Из этого утверждения следует получившая широкую известность лемма Аносова о замыкании, показывающая, как (локальное) условие гиперболичности и (нелокальное) условие возвращаемости движений приводят к существованию периодических движений в системе. Быстрому распространению этих новых идей и результатов немало способствовало то, что В. И. Арнольд и Я. Г. Синай, будучи участниками Международного конгресса математиков в Стокгольме (1962 г.), довели информацию о грубости геодезических потоков до сведения зарубежных коллег.

Во второй докладовской заметке Д. В. Аносова [2] анонсированы результаты об эргодических свойствах геодезических потоков на компактных римановых многообразиях отрицательной кривизны. В результате интегрирования сжимающегося и расширяющегося подрасслоений в фазовом пространстве указанных геодезических потоков получаются устойчивые и неустойчивые (орисферические) слоения, которые в трансверсальном направлении могут быть недифференцируемыми. В случае поверхностей, используя дифференцируемость орициклических слоений, Э. Хопф предложил способ 
доказательства эргодичности геодезических потоков. Д. В. Аносов открыл свойство устойчивых и неустойчивых слоений, которое позволяет использовать прием Хопфа в общем случае. Это свойство абсолютной непрерывности трансверсальных слоений, заключающееся в том, что биекции (вдоль неустойчивого слоения) близких площадок, расположенных на устойчивых слоях, абсолютно непрерывны по отношению к мере Лебега.

Результаты Д. В. Аносова о грубости и эргодических свойствах геодезических потоков, обладающих свойством гиперболичности, легли в основу его докторской диссертации, защищенной в МИАН в 1965 г. Топологические и статистические свойства динамических систем, удовлетворяющих условию гиперболичности (их первоначальное название: У-системы), составили содержание его секционного доклада на Международном конгрессе математиков в Москве (1966 г.). В недавно опубликованном интервью (см. [40]) на вопрос интервьюера о московском конгрессе Дмитрий Викторович отвечает: “Для меня, конечно, очень важным оказался контакт со Смейлом, который, по-видимому, впервые в развернутом виде изложил общую концепцию равномерной гиперболичности". В 1967 г. отдельным томом в Трудах МИАН вышла фундаментальная монография Д. В. Аносова об У-системах [3]. Вскоре Смейл [47] окрестил их (диффеоморфизмы и потоки) системами Аносова и это название прижилось. Дальнейшее развитие теории Аносова отчасти отражено, например, в [16].

Предложенной Д. В. Аносовым формализации идеи о глобальной гиперболичности, по его собственному признанию, весьма способствовало хорошее знакомство с классическими работами А. М. Ляпунова и О. Перрона об условной устойчивости для систем дифференциальных уравнений с переменными коэффициентами. По поводу влияния работ Н. Н. Боголюбова (относящихся к обоснованию метода усреднения) на формирование идеи о семействах устойчивых и неустойчивых подмногообразий фазового пространства, связанных с гиперболичностью, см. авторские реминисценции в [18]. Интересно, что в статье Д. В. Аносова о предельных циклах впервые появилось в явном виде инвариантное разложение касательных пространств в прямую сумму сжимающегося и расширяющегося подпространств $V_{+}, V_{-}$и (в случае потока) подпространства, порожденного вектором фазовой скорости, причем расслоения $V_{+}$и $V_{-}$ оказываются интегрируемыми.

Истоки современного взгляда на динамические системы с конечномерным фазовым пространством, возникающие в классической механике и других разделах физики, восходят к работам А. Пуанкаре конца XIX в. Фундаментальный прогресс, приведший к синтезу различных направлений в динамике и заложивший основы современной теории динамических систем, произошел в пятидесятых-шестидесятых годах XX в. Его основные элементы - это два открытия А. Н. Колмогорова (сохранение условно-периодических движений и энтропия в динамике) и гиперболическая революция, как ее назвал Дмитрий Викторович в своем обзоре [31].

Основные участники этой революции пришли из различных областей математики: С. Смейл - из топологии, Д. В. Аносов - из теории дифференциальных уравнений, Я.Г. Синай - из теории вероятностей, В.М.Алексеев - из небесной механики. Первый импульс, конечно, был дан Смейлом. Вскоре лидерство в гиперболической революции перешло к группе московских математиков (Алексеев, Аносов, Синай; В.И. Арнольд также проявлял живой интерес к новым идеям). Причина этого состояла в том, что московские математики принесли в эту новую область глубокое понимание и интуицию из различных разделов анализа, дифференциальных уравнений и теории вероятностей. Теория систем с максимально неустойчивым поведением траекторий послужила прообразом ряда новых направлений в теории динамических систем и привела к постановке интересных проблем, среди которых одной из важнейших остается задача глобальной топологической классификации диффеоморфизмов Аносова. Влияние работ Д. В. Аносова, одного из творцов гиперболической революции, на дальнейшее развитие динамики, несомненно, найдет отражение 
в будущих обзорах. Здесь отметим только то, что различные варианты ослабления свойства гиперболичности оказались плодотворными как в самой динамике (см., например, [44], [46]), так и в топологии, где введенные У. Тёрстоном псевдоаносовские гомеоморфизмы [48] в значительной степени прояснили классификацию с точностью до изотопии гомеоморфизмов поверхностей, данную Я. Нильсеном еще в 1930-е годы.

Д. В. Аносов применил свойства трансверсальных слоений к выводу своей знаменитой альтернативы: поток Аносова с интегральным инвариантом либо имеет непрерывный спектр и обладает K-свойством ${ }^{1}$ (а стало быть, и счетнократным лебеговским спектром), либо оказывается надстройкой (с постоянным временем возвращения) над диффеоморфизмом Аносова. Важным достижением является работа [4], в которой предложен гладкий вариант метода аппроксимаций периодическими преобразованиями. Этот метод позволил построить примеры гладких динамических систем с неожиданными спектральными свойствами; существенно то, что фазовые пространства этих систем суть компактные многообразия, допускающие нетривиальное действие окружности, в частности имеющее изолированные периодические точки.

Исходная глубоко оригинальная идея предложенного метода, не имеющего аналогов ни в динамике, ни в анализе, принадлежит Д. В. Аносову. Эта конструкция в дальнейшем совершенствовалась и к настоящему времени стала основным средством исследования так называемых лиувиллевых феноменов в динамике. В [5] Д. В. Аносов доказал, что на любом римановом многообразии размерности, большей двух, существует эргодический поток, сохраняющий объем.

С середины 1970-х годов Д. В. Аносов начинает новый тополого-геометрический цикл исследований. Им предложено доказательство (набросок см. в [6]) основного результата о римановых и финслеровых метриках, для которых соответствующий геодезический поток равномерно гиперболичен [11]. А именно, для этого требуется отсутствие сопряженных точек, негомотопность замкнутых геодезических нулю и между собой, а также равенство нулю их вариационного индекса. Затем Д. В. Аносов становится одним из основных разработчиков вопроса о существовании замкнутых несамопересекающихся геодезических на сферах. В начале 1980-х годов появляются его публикации [7] и [8], относящиеся к вариационной теории таких геодезических. Их цель - исправление доказательств С. Альбера и В. Клингенберга, касающихся существования несамопересекающихся замкнутых геодезических на сфере при условии так называемого защемления кривизны с коэффициентом $1 / 4$. Подход к этой задаче, пропагандировавшийся Клингенбергом, основан на введении структуры гильбертова многообразия в пространстве абсолютно непрерывных петель. Д. В. Аносову удалось установить ряд ключевых фактов об этом пространстве.

В [7] доказано, что 1) гладкое отображение многообразий задает гладкое отображение соответствующих многообразий петель и 2) ретракция пространства параметризованных петель, ставящая в соответствие петле ее же, но взятую с натуральным параметром, является непрерывным отображением, гомотопным тождественному, причем гомотопию можно выбрать с сохранением орбит естественного действия $\mathbb{S}^{1}$ и, следовательно, опустить до гомотопии соответствующих факторпространств. В [8] доказана теорема о гомологической нетривиальности вложения пространства больших окружностей $n$-сферы в соответствующее многообразие петель. В статье, озаглавленной "О типичных свойствах замкнутых геодезических" [9], приведено полное доказательство теоремы Абрагама о $C^{r}$-типичности $(r \geqslant 2)$ так называемых "бугристых метрик" (т. е. метрик, у которых каждая замкнутая геодезическая невырождена), что позволило строго оформить набросок Пуанкаре бифуркационного подхода к доказательству существования несамопересекающихся геодезических для всех римановых метрик положительной кривизны на $\mathbb{S}^{2}$.

Внимание топологов и геометров привлекла работа Д. В. Аносова [10] о связи гомологического и гомотопического подходов в теории неподвижных точек. В этой

\footnotetext{
1Это введенное Колмогоровым свойство квазирегулярности случайного процесса.
} 
работе усилен полученный в [39] результат о совпадении (с точностью до знака) чисел Нильсена и Лефшеца для отображений торов. Напомним, что число Нильсена отображения $f$ дает нижнюю оценку для числа неподвижных точек и по определению равно числу классов эквивалентности (неподвижных точек) с ненулевым индексом; точки $x_{1}, x_{2} \in \operatorname{Fix} f, x_{1} \neq x_{2}$, эквивалентны, если найдется путь $\gamma$ из $x_{1}$ в $x_{2}$, гомотопный (с закрепленными концами) пути $f \circ \gamma$. Обобщение результата из [39] на случай компактных нильмногообразий, установленное Д. В. Аносовым в [10], оказало стимулирующее влияние на современные исследования по числам Нильсена и их роли в теории неподвижных точек и динамике.

Отметим направление геометрических исследований Д. В. Аносова, к которому он возвращался на протяжении 40 лет, а именно, выяснение того, как могут уходить в бесконечность плоские кривые, накрывающие несамопересекающиеся кривые на замкнутых поверхностях (см. [29]). В начале 1960-х годов его внимание привлекла высказанная в 1930-х годах А. Вейлем идея о том, что при изучении потоков на поверхностях полезно рассматривать соответствующие универсально накрывающие потоки и лежащие на абсолюте предельные точки их полутраекторий (обобщение чисел вращения Пуанкаре). В 1965 г. им был получен результат в этом направлении, который в окончательной формулировке (см. [13]) гласит: если множество состояний равновесия непрерывного потока на замкнутой поверхности $M$ неположительной эйлеровой характеристики стягиваемо (или поток вещественно аналитичен), то каждая полутраектория универсально накрывающего потока либо находится в компактной части плоскости, либо имеет асимптотическое направление. Дмитрий Викторович пришел к выводу, что построению нелокальной качественной теории потоков на поверхностях должно предшествовать изучение расположения на поверхностях незамкнутых кривых без самопересечений и исследование асимптотики их поднятий на универсальную накрывающую.

Развитие этой идеи Д. В. Аносова, а также самой гиперболической теории привело в работах нижегородских и обнинских математиков к топологической классификации транзитивных потоков и слоений, нетривиальных минимальных множеств потоков и слоений, топологической классификации каскадов с нетривиальными базисными множествами, в том числе с одномерными аттракторами и репеллерами. Здесь уместно отметить построение Р. В. Плыкиным одномерного гиперболического аттрактора диффеоморфизма двумерной сферы и данную им классификацию растягивающихся аттракторов коразмерности один [45], а также монографии [41], [49], посвященные классификации каскадов на многообразиях малой размерности.

Серия работ Д. В. Аносова посвящена 21-й проблеме Гильберта о фуксовых системах линейных дифференциальных уравнений. Он переизложил основную конструкцию из работы А.А. Болибруха, приведшей к отрицательному решению упомянутой проблемы и оказавшейся прорывом в аналитической теории дифференциальных уравнений. Произведенное Д. В. Аносовым упрощение и внесенные им усовершенствования сделали соответствующую конструкцию достоянием широких кругов математиков. В [14], [15] используется геометрический подход к задаче о построении фуксовой системы по заданной монодромии. Этот подход был предложен Х. Рёрлем (1957) и впоследствии развит П. Делинем; существо метода состоит в сведении вопроса о разрешимости рассматриваемой задачи к проверке тривиальности некоторого ассоциированного с монодромией расслоения на римановой сфере. Д. В. Аносов предложил усовершенствование этого метода, позволившее напрямую связать сечение упомянутого расслоения с соответствующими фуксовыми системами. С другой стороны, упрощения, предложенные им, касались доказательства условий приводимости монодромии регулярных и фуксовых систем в терминах компонент их решений. Расширенный и переработанный материал из [14] включен в монографию [17], написанную совместно с А.А. Болибрухом; в ней представлен детальный исторический 
анализ постановок 21-й проблемы Гильберта. Более простые контрпримеры к проблеме Римана-Гильберта, в которых отрицательный ответ не зависит от положения особых точек на сфере Римана, опубликован Д. В. Аносовым в препринте [19].

В последние годы Дмитрий Викторович стал снова интересоваться задачами, относящимися к теории равномерно гиперболических систем. Хорошо известно, что топологическая сопряженность гиперболических автоморфизмов двумерного тора, задаваемых матрицами $A_{1}$ и $A_{2}$, эквивалентна сопряженности этих матриц посредством матрицы из $\mathrm{GL}(2, \mathbb{Z})$. В препринте [33] получен ответ на следующий вопрос: каков инвариант матриц (в дополнение к характеристическому многочлену) при таких сопряжениях? Ответ дан в терминах периодической части разложения в цепную дробь наклона собственного вектора матрицы $A$ и соответствующего предмарковского разбиения (т. е. разбиения на два параллелограмма, возникающего в конструкции Адлера-Вейсса марковского разбиения для автоморфизмов тора).

Работы [34]-[38] восходят к вопросу, поставленному Д. В. Аносовым еще в середине 1960-х годов: верно ли, что каждое гиперболическое множество динамической системы можно расширить (в любой его окрестности) до локально максимального инвариантного множества? Контрпримеры опубликованы С. Кровизье (2001) и Т. Фишером (2006). Не являющиеся локально максимальными инвариантные гиперболические множества, обладающие указанным выше свойством, Аносов предложил называть локально предмаксимальными, в [34] он установил, что указанное свойство - внутреннее, а именно: если $\Lambda_{1}, \Lambda_{2}$ - инвариантные гиперболические множества диффеоморфизмов $f_{1}, f_{2}$ и ограничения диффеоморфизмов на эти множества топологически сопряжены, то из локальной предмаксимальности одного из них следует это же свойство другого. Результат работы [35] о локальной предмаксимальности относится к возможной размерности таких множеств. В опубликованных ранее примерах инвариантных гиперболических множеств, не являющихся локально предмаксимальными, они имеют размерности 1 и 2. Для нульмерных гиперболических множеств Д. В. Аносов установил альтернативу: либо локальная максимальность, либо локальная предмаксимальность.

Немало времени Д.В. Аносов уделял научному редактированию, написанию обзоров (см. [12], [16], [28]), историко-математическим исследованиям и преподаванию. Вот свидетельство из воспоминаний А. Д. Мышкиса [42]: “Дмитрий Викторович Аносов - один из наших наиболее выдающихся математиков...; помимо науки, он активно занимается проблемами преподавания математики в школе". Книги [20], [22], [25], [26], [30], [32] и статьи [21], [23], [24], [27], относящиеся к этому направлению его деятельности, написаны им за последние 15-20 лет в соответствии с известной апокрифической историей. К ученому приходит молодой человек и на вопрос мэтра, чем бы он хотел заниматься, отвечает - историей науки. На это последовал совет - пока вы молоды, попробуйте заняться самой наукой, а ее историей, написанием книг на эту тему и популяризацией лучше заниматься в почтенном возрасте.

В книге [30] предложен вывод законов Кеплера из принципов ньютоновской механики. Эта прекрасно написанная книга, по уровню изложения рассчитанная на любознательного школьника, помимо вывода самих законов, содержит много интересных и малоизвестных фактов и представляется образцовым изложением затронутого предмета. Чтобы написать подобные книги и статьи (особенно [21]), надо обладать исключительной эрудицией в области самой математики, а также знать и чувствовать соответствующую историческую эпоху. Требуемые для этого качества точнее выразил известный историк математики Жюль Таннери - "нужно иметь вкус к истории, нужно понимать суть истории, иными словами, нужен интеллектуальный уровень, позволяющий чувствовать прошедшие эпохи как современные". Так, небольшая 
по объему статья [23] о работе А. Пуанкаре "О проблеме трех тел и об уравнениях динамики", удостоенной премии шведского короля Оскара II, дает живую панораму математической жизни конца XIX в. История этой работы, обнаруженная в ней знаменитая ошибка и ее исправление самим Пуанкаре, приведшие к открытию гомоклинических точек, совершенно уникальны. Статья включает и обзор результатов, связанных с этой тематикой, вплоть до самых последних работ. Ряд публикаций Дмитрия Викторовича относится к нашей недавней истории. Одна из них посвящена памяти известного математика Н. Н. Меймана [24]. В ней Д. В. изложил вклад Меймана в решение проблемы Рауса-Гурвица и со свойственным ему блеском набросал исторический очерк исследований в этом направлении, начиная с мемуара Максвелла о регуляторах (“On Governors”, 1868). Дмитрий Викторович завершает работу анализом связи результатов Меймана с математическим творчеством своего учителя Л. С. Понтрягина. Отметим здесь также статью [27] об Андрее Андреевиче Болибрухе, одном из ближайших коллег Д. В.

Что касается популяризации науки, поучителен предложенный Д. В. Аносовым в аспирантские годы пример, показывающий, что весьма ограничительные предположения теоремы Понтрягина об оптимальности (аналог принципа максимума для дифференциальных игр) отражают существо дела. Если в плоской задаче преследования управлением для преследователя служит произвольное ограниченное по модулю ускорение, а управлением для преследуемого - произвольная ограниченная по модулю скорость, то точная поимка оказывается невозможной. Объясняя этот пример, Дмитрий Викторович напомнил сказку о мальчике и крокодиле: крокодил может набрать сколь угодно большую скорость, но он не способен быстро ее менять, а мальчик, быстро меняя скорость, уворачивается от крокодила. В сказке крокодил заплакал горькими крокодиловыми слезами, жалуясь: “Это нечестно! Я же умею бегать быстрее!", а мальчик только смеялся.

Много сил Д. В. Аносов отдавал научно-организационной работе, педагогической и общественной активности. Он был членом Бюро Отделения математики РАН, заведовал лабораторией "Динамические системы”, отделом дифференциальных уравнений в МИАН и кафедрой теории динамических систем в МГУ, возглавлял диссертационный совет при МИАН, был членом Экспертного совета по математике и механике Высшей аттестационной комиссии, избирался в правление и вице-президентом Московского математического общества, принимал участие в деятельности попечительского совета МЦНМО, был председателем Экспертного совета РФФИ по математике, механике и информатике, участвовал в работе редколлегий ряда ведущих математических журналов, был председателем Оргкомитета Московской математической олимпиады.

Научные достижения Д.В. Аносова отмечены премией Московского математического общества (1965), Государственной премией СССР (1976), избранием членом-корреспондентом АН СССР (1990) и академиком РАН (1992), присуждением премии им. А. М. Ляпунова Российской академии наук (2001).

Работы Дмитрия Викторовича навсегда останутся в математике. Те, кто знали его, будут помнить этого замечательного человека и выдающегося математика.

С. М. Асеев, В.М. Бухитабер, Р.И. Григорчук, В.З. Гринес, Б. М. Гуревич, А.А. Давыдов, А.Ю. Жиров, Е. В. Жужома, М.И. Зеликин, А.Б. Каток, А.В. Клименко, В.В. Козлов, В.П. Лексин, М.И. Монастырский, А.И. Нейштадт, С.П. Новиков, Е.А. Сатаев, Я.Г. Синай, А. М. Стёпин 


\section{Цитированные работы Д. В. Аносова}

[1] "Грубость геодезических потоков на компактных римановых многообразиях отрицательной кривизны", Докл. АН СССР, 145:4 (1962), 707-709; англ. пер.: "Roughness of geodesic flows on compact Riemannian manifolds of negative curvature", Soviet Math. Dokl., 3 (1962), 1068-1070.

[2] "Эргодические свойства геодезических потоков на замкнутых многообразиях отрицательной кривизны", Докл. АН СССР, 151:6 (1963), 1250-1252; англ. пер.: "Ergodic properties of geodesic flows on closed Riemannian manifolds of negative curvature", Soviet Math. Dokl., 4 (1963), 1153-1156.

[3] "Геодезические потоки на замкнутых римановых многообразиях отрицательной кривизны", Тр. МИАН СССР, 90, 1967, 3-210; англ. пер.: "Geodesic flows on closed Riemann manifolds with negative curvature", Proc. Steklov Inst. Math., 90 (1967), 1-235.

[4] "Новые примеры в гладкой эргодической теории. Эргодические диффеоморфизмы", Тр. МMO, 23, 1970, 3-36 (совм. с А. Б. Катком); англ. пер.: "New examples in smooth ergodic theory. Ergodic diffeomorphisms", Trans. Mosc. Math. Soc., 23 (1970), 1-35 (with A. B. Katok).

[5] "Существование гладких эргодических потоков на гладких многообразиях", Изв. АН СССР. Сер. матем., 38:3 (1974), 518-545; англ. пер.: "Existence of smooth ergodic flows on smooth manifolds", Math. USSR-Izv., 8:3 (1974), 525-552.

[6] "Геодезические в финслеровой геометрии", Proceedings of the International Congress of Mathematicians (Vancouver, BC, 1974), v. 2, Canad. Math. Congress, Montreal, QC, 1975, 293-297.

[7] "Некоторые гомотопии в пространстве замкнутых кривых", Изв. АН СССР. Сер. матем., 44:6 (1980), 1219-1254; англ. пер.: "Certain homotopies in the space of closed curves", Math. USSR-Izv., 17:3 (1981), 423-453.

[8] "Некоторые гомологии в пространстве замкнутых кривых на $n$-мерной сфере", Изв. АН СССР. Сер. матем., 45:3 (1981), 467-490; англ. пер.: "Some homology classes in the space of closed curves in the n-dimensional sphere", Math. USSR-Izv., 18:3 (1982), 403-422.

[9] "О типичных свойствах замкнутых геодезических", Изв. АН СССР. Сер. матем., 46:4 (1982), 675-709; англ. пер.: "On generic properties of closed geodesics", Math. USSR-Izv., 21:1 (1983), 1-29.

[10] "О числах Нильсена отображений нильмногообразий”, УМH, 40:4(244) (1985), 133-134; англ. пер.: "The Nielsen numbers of maps of nil-manifolds", Russian Math. Surveys, 40:4 (1985), 149-150.

[11] “О геодезических потоках, удовлетворяющих условию ( $У)$ ", Современные проблемы математики. Математический анализ, алгебра, топология, Сборник статей. Посвящается академику Льву Семеновичу Понтрягину к его семидесятипятилетию, Тр. МИАН СССР, 167, 1985, 3-24; англ. пер.: "On geodesic flows satisfying condition (U)", Proc. Steklov Inst. Math., 167 (1986), 1-22.

[12] Динамические системы - 1, Итоги науки и техн. Сер. Соврем. пробл. матем. Фундам. направления, 1, ВИНИТИ, М., 1985, 244 с. (совм. с С.Х. Арансоном, В.И. Арнольдом, В. З. Гринесом, И. У. Бронштейном, Ю. С. Ильяшенко); англ. пер.: Ordinary differential equations and smooth dynamical systems, 3rd ed., Springer-Verlag, Berlin, 1997, vi+233 pp. (with S. Kh. Aranson, V.I. Arnold, I. U. Bronshtein, V.Z. Grines, Yu. S. Il'yashenko).

[13] "О поведении траекторий на плоскости Евклида или Лобачевского, накрывающих траектории потоков на замкнутых поверхностях. I", Изв. АН CCCP. Сер. матем., 51:1 (1987), 16-43; англ. пер.: "On the behavior in the Euclidean or Lobachevsky plane of trajectories that cover trajectories of flows on closed surfaces. I", Math. USSR-Izv., 30:1 (1988), 15-38. 
[14] Hilbert's 21st problem (according to Bolibruch), IMA preprint series № 660, Inst. Math. Appl., Univ. Minnesota, Minneapolis, MN, 1990, 35 pp.

http://www.ima.umn.edu/preprints/pp1990/660.pdf.

[15] An introduction to Hilbert's 21st problem, IMA preprint series № 861, Inst. Math. Appl., Univ. Minnesota, Minneapolis, MN, 1991, 59 pp.

http://www.ima.umn.edu/preprints/pp1991/861.pdf.

[16] “Динамические системы с гиперболическим поведением”, Динамические системъь - 9, Итоги науки и техн. Сер. Соврем. пробл. матем. Фундам. направления, 66, ВИНИТИ, М., 1991, 5-242 (совм. с С.Х. Арансоном, В.З. Гринесом, Р. В. Плыкиным, Е. А. Сатаевым, А. В. Сафоновым, В. В. Солодовым, А. Н. Старковым, А. М. Степиным, С. В. Шлячковым); англ. пер.: Dynamical systems IX, Encyclopaedia Math. Sci., 66, Springer, Berlin, 1995, 235 pp. (with S. Kh. Aranson, V. Z. Grines, R. V. Plykin, E. A. Sataev, A. V. Safonov, V. V. Solodov, A. N. Starkov, A. M. Stepin, S. V. Shlyachkov).

[17] The Riemann-Hilbert problem, Aspects Math., 22, Friedr. Vieweg \& Sohn, Braunschweig, 1994, x+190 pp. (with A. A. Bolibruch).

[18] "О вкладе Н.Н. Боголюбова в теорию динамических систем", УМН, 49:5(299) (1994), 5-20; англ. пер.: "On the contribution of N. N. Bogolyubov to the theory of dynamical systems", Russian Math. Surveys, 49:5 (1994), 1-18.

[19] A simple counterexample to the Fuchsian version of the Hilbert 21st problem, preprint № 124, Max-Planck-Institut für Mathematik, Bonn, 1998, 11 pp.

http://webdoc.sub.gwdg.de/ebook/serien/e/mpi_mathematik/1998/124.ps.

[20] Лекиии по линейной алгебре, Регулярная и хаотическая динамика, М., 1999, 105 с.

[21] "К истории вывода законов Кеплера из законов механики", Истор.-матем. исслед., сер. 2, 5(40), Изд-во "Янус-К”, М., 2000, 9-25.

[22] Взгляд на математику и нечто из нее, МЦНМО, М., 2000, 32 с.

[23] "Пуанкаре и проблемы Оскара II", Истор.-матем. исслед., сер. 2, 6(41), Изд-во "Янус-К", М., 2001, 57-72.

[24] "Нас осталось так мало...", Истор.-матем. исслед., сер. 2, 7(42), Изд-во "Янус-К", М., 2002, 166-189 (совм. с М. И. Монастырским, М. А. Соловьевым).

[25] О спектралъных кратностях в эргодической теории, Совр. пробл. матем., 3, МИАН, М., 2003, 86 c.

[26] Отображения окружности, векторные поля и их приложения, МЦНМО, М., 2003, 120 c.

[27] “Андрей Андреевич Болибрух в жизни и науке (30.01.1950-11.11.2003)", УМН, 59:6(360) (2004), 3-22 (совм. с В.П. Лексиным); англ. пер.: "Andrei Andreevich Bolibrukh in life and science (30 January 1950-11 November 2003)", Russian Math. Surveys, 59:6 (2004), 1009-1028 (with V. P. Leksin).

[28] "On the development of the theory of dynamical systems during the past quarter century", Surveys in modern mathematics, London Math. Soc. Lecture Note Ser., 321, Cambridge Univ. Press, Cambridge, 2005, 70-185.

[29] "Нелокальное асимптотическое поведение кривых и слоев ламинаций на универсальных накрывающих", Тр. МИАН, 249, Наука, М., 2005, 3-239 (совм. с Е. В. Жужомой); англ. пер.: "Nonlocal asymptotic behavior of curves and leaves of laminations on universal coverings", Proc. Steklov Inst. Math., 249 (2005), 1-221 (with E. V. Zhuzhoma).

[30] От Нъютона к Кеплеру, МЦНМО, М., 2006, 272 с.

[31] “Динамические системы в 60-е годы: гиперболическая революция", Maтематические события XX века, Фазис, М., 2003, 1-18 с.; англ. пер.: "Dynamical systems in the 1960s: The hyperbolic revolution", Mathematical events of the twentieth century, Springer, Berlin, 2006, 1-17. 
[32] Дифференциальные уравнения: то решаем, то рисуем, МЦНМО, М., 2008, 200 с.

[33] On the hyperbolic automorphisms of the 2-torus and their Markov partions, preprint № 54, Max-Planck-Institut für Mathematik, Bonn, 2008, 34 pp., http://webdoc.sub.gwdg.de/ebook/serien/e/mpi_mathematik/2008/54.pdf, arXiv: 0810.5269 (with A. V. Klimenko, G. Kolutsky).

[34] "Intrinsic character of one property of hyperbolic sets", J. Dyn. Control Syst., 16:4 (2010), 485-493.

[35] "Расширение нульмерных гиперболических множеств до локально максимальных", Матем. сб., 201:7 (2010), 3-14; англ. пер.: "Extension of zero-dimensional hyperbolic sets to locally maximal ones", Sb. Math., 201:7 (2010), 935-946.

[36] "О некоторых гиперболических множествах", Матем. заметки, 87:5 (2010), 650-668; англ. пер.: "On certain hyperbolic sets", Math. Notes, 87:5 (2010), 608-622.

[37] “Локальная максимальность гиперболических множеств", Современные проблемъ математики, Сборник статей. К 75-летию Института, Тр. МИАН, 273, МАИК, М., 2011, 28-29; англ. пер.: "Local maximality of hyperbolic sets", Proc. Steklov Inst. Math., 273:1 (2011), 23-24.

[38] "О траекториях, целиком расположенных возле гиперболического множества", Труди Шестой Международной конференции по дифференциальным и функиионально-дифференииальным уравнениям (Москва, 14-21 августа, 2011). Часть 1, СМФН, 45, РУДН, М., 2012, 5-17; англ. пер.: "On trajectories entirely situated near a hyperbolic set", J. Math. Sci. (N. Y.), 201:5 (2014), 553-565.

\section{Цитированные работы других авторов}

[39] R. B. S. Brooks, R. F. Brown, J. Pak, D. H. Taylor, "Nielsen numbers of maps of tori", Proc. Amer. Math. Soc., 52:1 (1975), 398-400.

[40] В. Б. Демидович, $K$ истории мехмата МГУ, Изд-во попечит. совета мех.-матем. факультета МГУ, М., 2013, 460 с.

[41] В. З. Гринес, О. В. Починка, Введение в топологическую классификачию каскадов на многообразиях размерности два и три, РХД, М.-Ижевск, 2011, 424 с.

[42] А.Д. Мышкис, Советские математики. Мои воспоминания, ЛКИ, М., 2007, $304 \mathrm{c}$.

[43] А.И. Нейштадт, "Усреднение, прохождение через резонансы и захват в резонанс в двухчастотных системах", УМH, 69:5(419) (2014), 3-80; англ. пер.: A. I. Neishtadt, "Averaging, passage through resonances, and capture into resonance in two-frequency systems", Russian Math. Surveys, 69:5 (2014), 771-843.

[44] Я.Б. Песин, "Характеристические показатели Ляпунова и гладкая эргодическая теория", УМH, 32:4(196) (1977), 55-112; англ. пер.: Ya. B. Pesin, "Characteristic Lyapunov exponents and smooth ergodic theory", Russian Math. Surveys, 32:4 (1977), 55-114.

[45] Р. В. Плыкин, "О геометрии гиперболических аттракторов гладких каскадов", УМH, 39:6(240) (1984), 75-113; англ. пер.: R. V. Plykin, "On the geometry of hyperbolic attractors of smooth cascades", Russian Math. Surveys, 39:6 (1984), 85-131.

[46] Е.А. Сатаев, "Инвариантные меры для сингулярно гиперболических аттракторов", Матем. сб., 201:3 (2010), 107-160; англ. пер.: Е. A. Sataev, "Invariant measures for singular hyperbolic attractors", Sb. Math., 201:3 (2010), 419-470.

[47] С. Смейл, “Дифференцируемые динамические системы”, УМН, 25:1(151) (1970), 113-185; пер. с англ.: S. Smale, "Differentiable dynamical systems", Bull. Amer. Math. Soc., 73:6 (1967), 747-817.

[48] W.P. Thurston, "On the geometry and dynamics of diffeomorphisms of surfaces", Bull. Amer. Math. Soc. (N. S.), 19:2 (1988), 417-431.

[49] А.Ю. Жиров, Топологическая сопряженность псевдоаносовских гомеоморфизмов, МЦНМО, М., 2013, 368 с. 